\title{
Enhanced effect of fibroblast growth factor-2-containing dalteparin/protamine nanoparticles on hair growth
}

This article was published in the following Dove Press journal:

Clinical, Cosmetic and Investigational Dermatology

17 May 2016

Number of times this article has been viewed

\author{
Yuki Takabayashi' \\ Masaki Nambu' \\ Masayuki Ishihara ${ }^{2}$ \\ Masahiro Kuwabara' \\ Koichi Fukuda ${ }^{2}$ \\ Shingo Nakamura ${ }^{2}$ \\ Hidemi Hattori ${ }^{2}$ \\ Tomoharu Kiyosawa' \\ 'Department of Plastic and \\ Reconstructive Surgery, ${ }^{2}$ Division of \\ Biomedical Engineering, Research \\ Institute, National Defense Medical \\ College, Tokorozawa, Saitama, Japan
}

Correspondence: Masayuki Ishihara Division of Biomedical Engineering, Research Institute, National Defense Medical College, 3-2 Namiki, Tokorozawa 359-85I3, Saitama, Japan

$\mathrm{Tel}+8|42995| 60 \mid$

Fax +81 429911611

Email ishihara@ndmc.ac.jp
Purpose: Although treatments for alopecia are in high demand, not all treatments are safe and reliable. Dalteparin/protamine nanoparticles (D/P NPs) can effectively carry growth factors (GFs) such as fibroblast GF (FGF)-2. The purpose of this study was to identify the effects of FGF-2-containing D/P NPs (FGF-2\&D/P NPs) on hair growth.

Patients and methods: In this study, the participants were 12 volunteers with thin hair. One milliliter of FGF-2 $(100 \mathrm{ng} / \mathrm{mL})$ and D/P NPs $(56 \mu \mathrm{g} / \mathrm{mL})$ was applied and massaged on the skin of the scalp by the participants twice a day. They were evaluated for 6 months. Participants were photographed using a digital camera for general observation and a hair diagnosis system for measuring hair diameter.

Results: The mean diameter of the hairs was significantly higher following the application of FGF-2\&D/P NPs for 6 months. Objective improvements in thin hair were observed in two cases. Nine participants experienced greater bounce and hair resilience.

Conclusion: The transdermal application of FGF-2\&D/P NPs to the scalp can be used as a new treatment for alopecia.

Keywords: hair growth, dalteparin/protamine nanoparticles, fibroblast growth factor, transdermal application

\section{Introduction}

Hair loss is not a life-threatening event, and the number of patients suffering from hair loss has dramatically increased. Hair loss affects the social interactions and the psychological well-being of the patient. Currently, two anti-hair loss drugs, ie, finasteride and minoxidil, are available. These drugs have been used clinically, but their effect is reported to be limited, transient, and somewhat unpredictable. ${ }^{1}$ Therefore, it is important to develop an effective pharmacological treatment for hair loss.

Hair growth is a complex and cyclically controlled process categorized by the following three phases: anagen, a finite period of hair fiber production; catagen, a brief regression phase; and telogen, a resting phase. ${ }^{2,3}$ The precise mechanism regulating the hair growth cycle has not been fully characterized. However, it is well known that both the continuation and transition of each phase result from the elaborate interactions between mesenchymal dermal papillae (DP) and adjacent follicular epithelium, the matrix, and the outer root sheath. ${ }^{3,4}$ Particularly, the DP cells located at the base of the hair follicle and surrounded by the matrix indicated the potential to induce hair follicle formation. ${ }^{5,6}$ The hair follicles, including DP cells, are organs, which are responsible for the production of hair fibers by inducing and maintaining the anagen 
phase. Various cytokines and growth factors (GFs) are involved in the regulation of hair follicles and hair growth cycle. Specifically, fibroblast GF (FGF)-2 has been shown to promote DP cell proliferation and increase the hair follicle size in mice. ${ }^{6,7}$ FGF-2 along with other GFs has been reported to have a protective effect on neomycin-induced hair loss. ${ }^{8}$ A study on the controlled release of FGF-2 has also demonstrated that hydrogel enabled FGF-2 to positively act on the hair growth cycle of mice. ${ }^{9}$

Polyelectrolyte complexes (PECs) are generated by electrostatic interactions between oppositely charged polyelectrolytes, that is, dalteparin (low-molecular-weight heparin) and protamine). Nonstoichiometric PECs are produced when this interaction occurs at nonequivalent ratios. This causes each PEC particle to carry an excess charge..$^{10,11}$ Proteins interact with both synthetic and natural PECs. ${ }^{12}$ Heparin and dalteparin specifically interact with functional proteins, including GFs, cytokines, extracellular matrix components, and adhesion molecules, with high affinity. ${ }^{13,14}$ Thus, heparin may be useful as a therapeutic agent in various pathological conditions involving functional proteins. However, high-dose heparin cannot be used on account of high risk of bleeding. ${ }^{15}$ In contrast, dalteparin ( 5,000 Da) has pharmacological and practical advantages compared to heparin. The lower protein-binding activity of dalteparin produces a low, stable, and predictable anticoagulant response. This bypasses the need for laboratory monitoring of drug levels to adjust the dosage. ${ }^{16}$ In addition, given the longer plasma half-life, one or two subcutaneous injections per day were sufficient to maintain therapeutic concentrations. ${ }^{16}$ Conversely, protamine, a purified mixture of proteins, was obtained from fish sperm, neutralized heparin, and dalteparin by forming a stable complex that lacked anticoagulant activity. ${ }^{16}$ Protamine was also employed in clinical use to reverse the anticoagulant activity of heparin following cardiopulmonary bypass. It was also used in cases of heparin-induced bleeding. ${ }^{17}$ In this article, we reported about dalteparin/protamine nanoparticles $(\mathrm{D} / \mathrm{P}$ NPs), which were originally prepared as PECs. ${ }^{18,19}$ The D/P NPs were specifically bound to various heparin-binding GFs such as FGF-2, ${ }^{20,21}$ hepatocyte growth factor, ${ }^{22}$ and other GFs in platelet-rich plasma (PRP). ${ }^{23} \mathrm{FGF}-2 \& \mathrm{D} / \mathrm{P}$ NPs substantially induced vascularization and fibrous tissue formation. This was because of the stabilization, activation, and gradual release of FGF-2 molecules from FGF-2\&D/P NPs as effective microcarriers for FGF-2. ${ }^{20,21}$ The D/P NPs functioned as a carrier for the controlled release of GFs secreted from activated PRP. The GFs from PRP-containing D/P NPs (GFs from PRP\&D/P NPs) also exhibited a substantial ability to induce vascularization and fibrous tissue formations when locally administrated in vivo. ${ }^{23}$ A clinical study using GFs from PRP\&D/P NPs demonstrated that local injections of autologous GFs from PRP\&D/P NPs facilitated hair growth as a new treatment for alopecia. ${ }^{24}$ However, because the treatment was extremely painful, the transdermal application of an effective hair grower to the scalp is preferable. In this study, a basic and clinical experiment was performed to evaluate whether the transdermal application of FGF-2\&D/P NPs ( 200-250 $\mathrm{nm}$ in diameter) to the scalp can be used as a new treatment for alopecia.

\section{Patients and methods Preparation of FGF-2\&D/P NPs}

The D/P NPs were prepared by modifying the method as described earlier. ${ }^{20,25}$ To summarize, $2.5 \mathrm{~mL}$ of dalteparin solution (Fragmin: $6.4 \mathrm{mg} / \mathrm{mL}$; Kissei Pharmaceutical Co., Tokyo, Japan), followed by $1.2 \mathrm{~mL}$ of protamine solution (10 mg/mL; Mochida Pharmaceutical Co., Tokyo, Japan) was added into $500 \mathrm{~mL}$ of saline (Otsuka Normal Saline; Otsuka Pharmaceutical Co., Ltd. Tokyo, Japan). The white solution was mixed vigorously. One milliliter of recombinant human FGF-2 (50 $\mu \mathrm{g} / \mathrm{mL}$; Fiblast; Kaken Pharmaceutical Co., Tokyo, Japan) was added to $500 \mathrm{~mL}$ of saline to produce diluted FGF-2\&D/P NPs. The final concentrations of FGF-2 and D/P NPs were estimated at $100 \mathrm{ng} / \mathrm{mL}$ and $56 \mu \mathrm{g} / \mathrm{mL}$, respectively. Under these conditions, almost all FGF-2 molecules were immobilized on the D/P NPs because $\sim 5 \mu$ of FGF-2 was immobilized on $1 \mathrm{mg}$ of D/P NPs..$^{11,17}$ The FGF-2\&D/P NPs were stored at $4{ }^{\circ} \mathrm{C}$.

\section{Measurement of particle size and zeta charge}

Particle size and zeta charge of FGF-2\&D/P NPs were measured by ELSZ-1000 (Otsuka Electronics Co. Ltd., Osaka, Japan). Weekly measurements were performed for 10 weeks. FGF-2\&D/P NPs were stored at $25^{\circ} \mathrm{C}$ (room temperature) for 10 weeks.

\section{Mitogenic activity of standalone FGF-2 and FGF-2\&D/P NPs on human microvascular endothelial cells}

In this study, FGF-2 (100 ng/mL), D/P NPs $(56 \mu \mathrm{g} / \mathrm{mL})$, and FGF-2 $(100 \mathrm{ng} / \mathrm{mL})$ and D/P NPs $(56 \mu \mathrm{g} / \mathrm{mL})$ were incubated either at $4^{\circ} \mathrm{C}$ or $37^{\circ} \mathrm{C}$ for 2 weeks and diluted with Dulbecco's Modified Eagle's Medium (DMEM) containing 5\% fetal bovine serum (FBS). Human microvascular endothelial cells (HMVECs) from Takara Bio Inc., Shiga, Japan manufactured 
by PromoCell GmbH, Heiderlerg, Germany were cultured in DMEM containing 5\% FBS, FGF-2 (5 ng/mL), and/or D/P NPs $(2.8 \mu \mathrm{g} / \mathrm{mL}) .{ }^{18}$ The tissue used by PromoCell for the isolation of human cell cultures is derived from donors who have signed an informed consent form, which outlines in detail the purpose of the donation and the procedure for processing the tissue. The ethical committee in National Defense Medical College does not require approval in usage of commercially available human-derived cells (PromoCell). The cells used in these studies had cell-cycle passages ranging between four and eight. MVECs were seeded at an initial density of 3,000 cells/well in 96-well tissue culture plates. They were grown for 3 days in $200 \mu \mathrm{L}$ of DMEM containing 5\% FBS, $100 \mathrm{U} / \mathrm{mL}$ penicillin $\mathrm{G}, 100 \mu \mathrm{g} / \mathrm{mL}$ streptomycin, and either standalone FGF-2, or FGF-2\&D/P NPs, or standalone D/P NPs. Following the incubation, the medium was removed and $100 \mu \mathrm{L}$ of fresh medium containing $10 \mu \mathrm{L}$ of water-soluble tetrazolium salts-1 reagent (Cell Counting Kit; Dojindo Co. Ltd., Kumamoto, Japan) was added to each well. The cell number was estimated by measuring the absorbance at $450 \mathrm{~nm}$ using a microplate reader.

\section{The penetration of FGF-2\&D/P NPs in rat skin}

Inbred male Fischer 344 rats (Japan SLC, Inc., Shizuoka, Japan) weighing 170-190 g were used in all the experiments. All rats were maintained on a standard laboratory diet with water ad libitum. Rats were anesthetized by intraperitoneal injections of $0.15 \mathrm{mg} / \mathrm{kg}$ medetomidine, $2 \mathrm{mg} / \mathrm{kg}$ midazolam, and $5 \mathrm{mg} / \mathrm{kg}$ butorphanol tartrate. The dorsal skin hair was shaved and depilated using a depilatory cream. Intraperitoneal injections of $0.15 \mathrm{mg} / \mathrm{kg}$ atipamezole roused the rats from the anesthesia. FGF-2\&D/P NPs, standalone FGF-2, or saline (control) was applied to the dorsal skin twice daily for 3 days. In this experiment, each solution $(0.2 \mathrm{~mL})$ was rubbed into the skin, and then $0.1 \mathrm{~mL}$ of solution was in contact with the skin for 20 minutes or more to wake up from the anesthesia. On the third day, the rats were killed and their skin samples were taken.

Skin specimens were fixed in $10 \%$ formalin solution (Wako Pure Chemical Industries, Ltd., Osaka, Japan), which were embedded in paraffin and sectioned at $4 \mu \mathrm{m}$. The sections were mounted onto glass slides and stained with FGF-2 antibody, which was performed at Kyodo Byori Inc. (Hyogo, Japan). All animal experiments were approved and carried out following the approval and guidelines for animal experimentation set by the National Defense Medical College, Saitama, Japan.

\section{Participants of clinical research}

The participants were 12 individuals (eleven men, one woman) with ages ranging from 41 years to 77 years (mean age, 58.3 years) with thin hair and without any treatment of thin hair for at least 6 months. The participants visited the hospital monthly to receive inspections and bottles of FGF2\&D/P NPs. Participants were photographed using a digital camera for general observation and a hair diagnosis system (Folliscope; LeadM Corp, Seoul, South Korea) for measuring hair diameter. All research protocols were submitted and approved by the ethics committee of the National Defense Medical College (clinical trial No. 1148 and No. 1241) and registered in the University Hospital Medical Information Network Center (UMIN000011748). Each participant was informed of the protocols and signed a written consent form before participating in the study.

\section{The application and evaluation of FGF-2\&D/P NPs on the skin of the scalp}

One milliliter of FGF-2\&D/P NPs was applied and massaged on the skin of the scalp by participants twice a day. Monthly observations were conducted on the participants for 6 months. The Folliscope captured ten dermoscopic images at $1 \mathrm{~cm}$ intervals toward the front left from the parietal region. The diameters of all hair shafts in each dermoscopic image were measured. The mean diameter of all hairs and the percentage of terminal hair (with diameter $>0.040 \mathrm{~mm}$ ) were calculated using the measured data.

All values are expressed as mean $\pm \mathrm{SD}$. Statistical analysis was performed with Wilcoxon signed-rank test using JMP Pro 11.2.0 software (SAS Institute Inc., Cary, NC, USA). $P<0.05$ was considered statistically significant.

\section{Results \\ Measurement of particle size and zeta charge}

Measurements for particle size and zeta charge of FGF$2 \& \mathrm{D} / \mathrm{P} \mathrm{NPs}$ were performed every week for 10 weeks at $25^{\circ} \mathrm{C}$ (room temperature). The diameter of the FGF-2\&D/P NPs increased from $\sim 170 \mathrm{~nm}$ to $210 \mathrm{~nm}$ during the first 1-2 weeks, and thereafter it was stable $(\sim 210-220 \mathrm{~nm})$. The zeta charge was also stable ( $\sim 25 \mathrm{mV}$ to $-28 \mathrm{mV}$; Figure 1$)$. In addition, the morphology of the FGF-2\&D/P NPs was unchanged for 10 weeks at $25^{\circ} \mathrm{C}$ (data not shown). Thus, the FGF-2\&D/P NPs were stable in terms of particle size, zeta charge, and morphology for at least 10 weeks at $25^{\circ} \mathrm{C}$. 


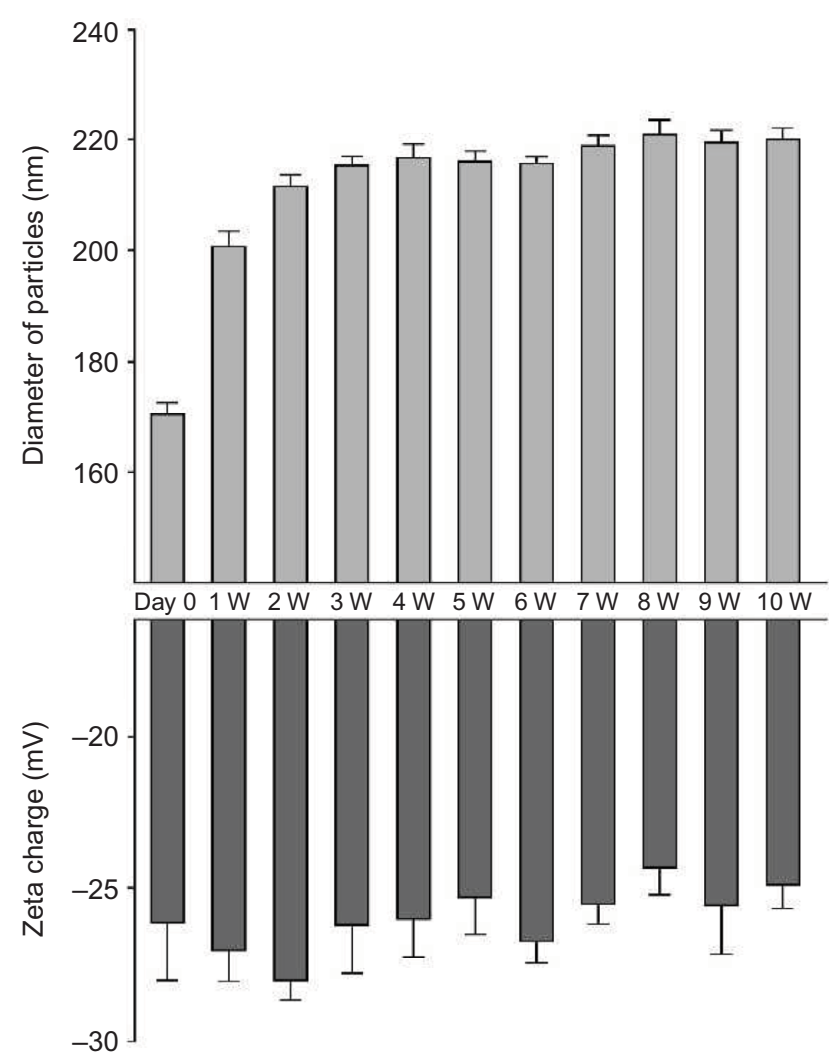

Figure I Diameter and zeta charge of FGF-2\&D/P NPs stored at room temperature. Notes: Measurements were performed weekly for 10 weeks. The diameter of FGF-2\&D/P NPs was stable ( 210-220 nm), and the zeta charge was also stable ( - $25 \mathrm{mV}$ to $-28 \mathrm{mV}$ ).

Abbreviations: D/P, dalteparin/protamine; FGF-2, fibroblast growth factor-2; NPs, nanoparticles; W, week.

\section{Mitogenic activity of standalone FGF-2 and FGF-2\&D/P NPs on HMVECs}

In this study, FGF-2, D/P NPs, and FGF-2\&D/P NPs were incubated at $4^{\circ} \mathrm{C}$ or $37^{\circ} \mathrm{C}$ for 2 weeks, and diluted with DMEM containing 5\% FBS. HMVECs were cultured and growth stimulated in DMEM containing 5\% FBS, FGF-2 (final concentration: $5 \mathrm{ng} / \mathrm{mL}$ ), and/or D/P NPs (final concentration: $2.8 \mu \mathrm{g} / \mathrm{mL}$ ). Standalone D/P NPs incubated at both $4^{\circ} \mathrm{C}$ and $37^{\circ} \mathrm{C}$ for 2 weeks showed no cell growthstimulatory effect. Although the FGF-2 without D/P NPs incubated at $37^{\circ} \mathrm{C}$ lost the cell growth-stimulatory effect, the FGF- 2 without D/P NPs at $4^{\circ} \mathrm{C}$ retained a partial cell growth-stimulatory effect. The FGF-2\&D/P NPs incubated at both $4^{\circ} \mathrm{C}$ and $37^{\circ} \mathrm{C}$ maintained the cell growth-stimulatory effect. Thus, D/P NPs have a stabilizing effect on FGF-2 bioactivity (Figure 2).

\section{Penetration of FGF-2\&D/P NPs in rat skin}

FGF-2\&D/P NPs, standalone FGF-2, or saline was applied to the dorsal skin without any complications. The dorsal skin showed no signs of acute inflammation, skin redness, or swelling during the experimental period (3 days). There were no differences in skin appearances between FGF-2\&D/P NPs, standalone FGF-2, and saline (control) conditions.
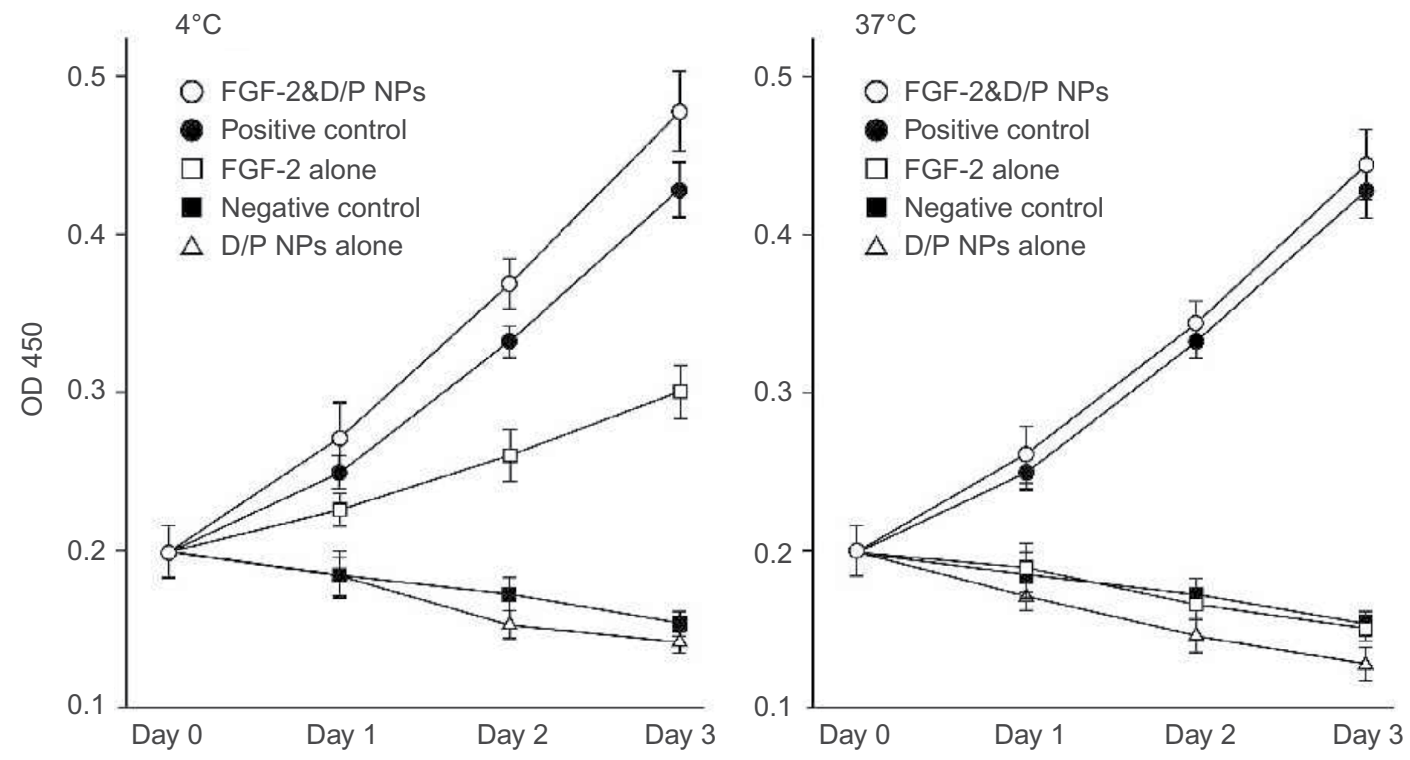

Figure 2 The protective effect of D/P NPs on FGF-2 bioactivity.

Notes: FGF-2, D/P NPs, and FGF-2\&D/P NPs were incubated at $4^{\circ} \mathrm{C}$ or $37^{\circ} \mathrm{C}$ for 2 weeks. The growth-stimulatory activity for HMVECs in vitro was stable for at least 2 weeks at $37^{\circ} \mathrm{C}$ compared to the growth-stimulatory activity for those without D/P NPs. Positive control implies fresh FGF-2. Negative control indicates the absence of FGF-2 and D/P NPs.

Abbreviations: D/P, dalteparin/protamine; FGF-2, fibroblast growth factor-2; HMVECs, human microvascular endothelial cells; NPs, nanoparticles; OD, optical density. 

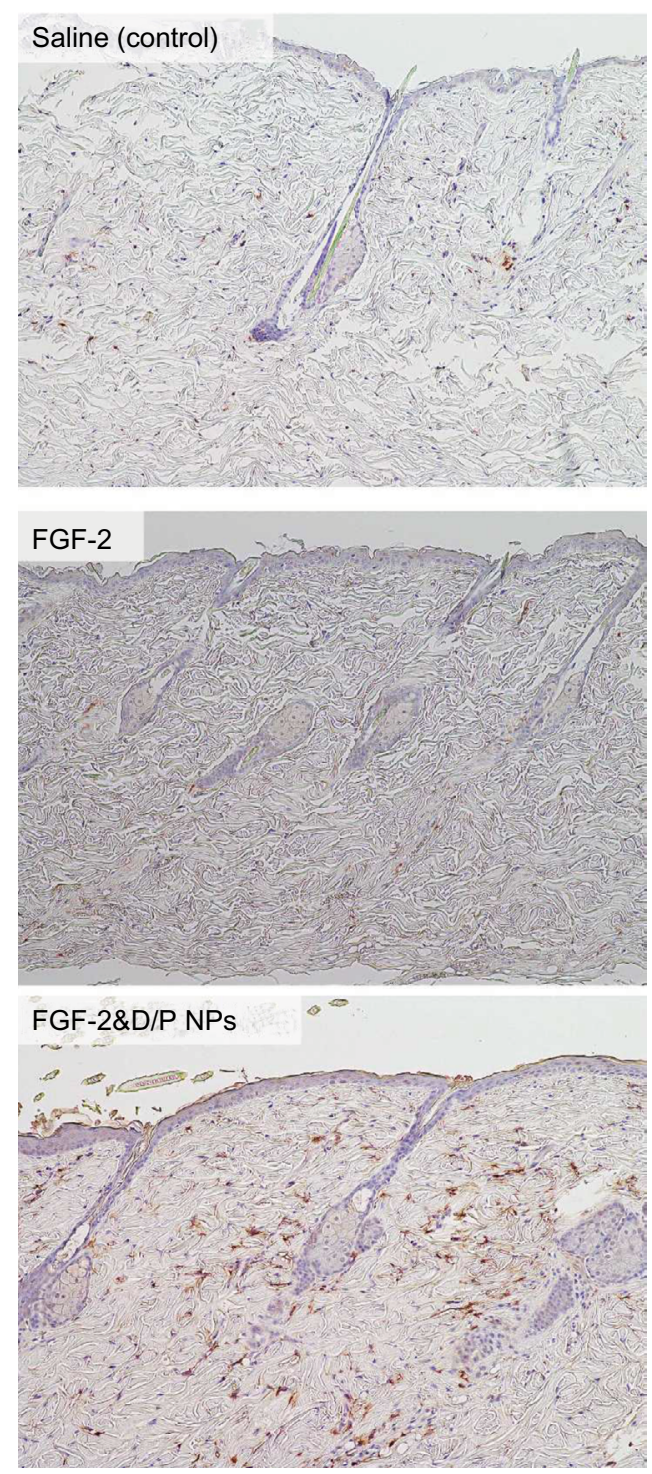

Figure 3 FGF-2 antibody staining of the F344 rat dorsal skin with applied saline (control), standalone FGF-2, or FGF-2\&D/P NPs.

Notes: The FGF-2\&D/P NPs-applied group was stained more strongly than FGF-2 and saline-applied groups.

Abbreviations: D/P, dalteparin/protamine; FGF-2, fibroblast growth factor-2; NPs, nanoparticles.

Immunohistological evaluations were performed to confirm the skin penetration of FGF-2\&D/P NPs. Figure 3 shows the representative microphotographs of the FGF-2 antibody staining for saline (control), standalone FGF-2, and FGF$2 \& \mathrm{D} / \mathrm{P}$ NPs applied to the dorsal skin on day 3 . The group that received FGF-2\&D/P NPs applications was stained more strongly than the group that received standalone FGF-2 and saline application (control). Hence, we concluded that FGF$2 \& \mathrm{D} / \mathrm{P}$ NPs had deeper penetration in rat skin.

\section{Application of FGF-2\&D/P NPs onto the skin of the scalp}

No signs of acute inflammation, allergic symptoms, skin redness, swelling, or itching were observed in the scalp skin during the experimental period (6 months). The mean diameter of the hairs was significantly higher following the application of FGF-2\&D/P NPs for 6 months $(0.0324 \pm 0.0035 \mathrm{~mm})$ than the mean hair diameter prior to the application of FGF-2\&D/P NPs $(0.0290 \pm 0.0047 \mathrm{~mm})$. The percentage of terminal hair was also significantly higher after the application $(26.4 \% \pm 11.6 \%)$ than before the application $(21.5 \% \pm 11.8 \%)$. Objective improvements in thin hair were observed in two cases (Figure 4). Nine participants experienced greater bounce and hair resilience. The remaining three participants did not observe any changes.

\section{Discussion}

Various cytokines and GFs are involved in the regulation of hair follicles and hair growth cycle. In particular, FGF-2 was found to act on hair follicles as a regulator of hair growth cycle. ${ }^{26-28}$ However, when GFs are directly applied to the scalp, the local biological activity of FGF-2 may be limited in vivo by the rapid clearance. Furthermore, it is unlikely that direct application of GFs to the scalp penetrates the pores and hair follicles, since FGF-2 molecule is aqueously dissolved through hydration reaction.

In this article, we reported about D/P NPs, which were originally prepared as PECs. ${ }^{18}$ The D/P NPs were specifically bound to FGF-2, ${ }^{20,21}$ hepatocyte growth factor, ${ }^{22}$ and other GFs in PRP. ${ }^{23,24}$ The local injection of D/P NPs provided an excellent carrier that immobilized, retained, and gradually released various GFs from the PRP for the induction of hair follicles. ${ }^{24}$ The local injection of GFs from PRP\&D/P NPs also had a substantial ability to enhance vascularization and fibrous tissue formation, ${ }^{23}$ flap survival rate,${ }^{29}$ and healing in split-thickness skin graft donor sites. ${ }^{30}$ Results of the clinical study on the injection of GFs from PRP\&D/P NPs indicated significant improvements in hair thickness following localized injections (at least five times). However, the treatment was extremely painful. ${ }^{24}$ Thus, the development of a reliable, efficient, and safe transdermal FGF-2 delivery system using D/P NPs may present potential applications for hair growth.

The D/P NPs actually protect FGF-2 from heat and proteolytic inactivation and enhance their biological activities. ${ }^{21}$ In addition, FGF-2 immobilized onto D/P NPs was released with a half release time of $\sim 7$ days in vitro. ${ }^{20}$ Typically, NPs tend to be assemble with each other and grow to form larger micro-assemblies. This study showed that the diameter of FGF-2\&D/P NPs was stable $(\sim 200-250 \mathrm{~nm})$, and that the zeta charge was also stable ( $-25 \mathrm{mV}$ to $-28 \mathrm{mV}$; Figure 1). Furthermore, the morphology of FGF-2\&D/P NPs (data not shown) and the growth-stimulatory activity for HMVECs in vitro (Figure 2) were stable for at least 2 weeks at $37^{\circ} \mathrm{C}$ 
A
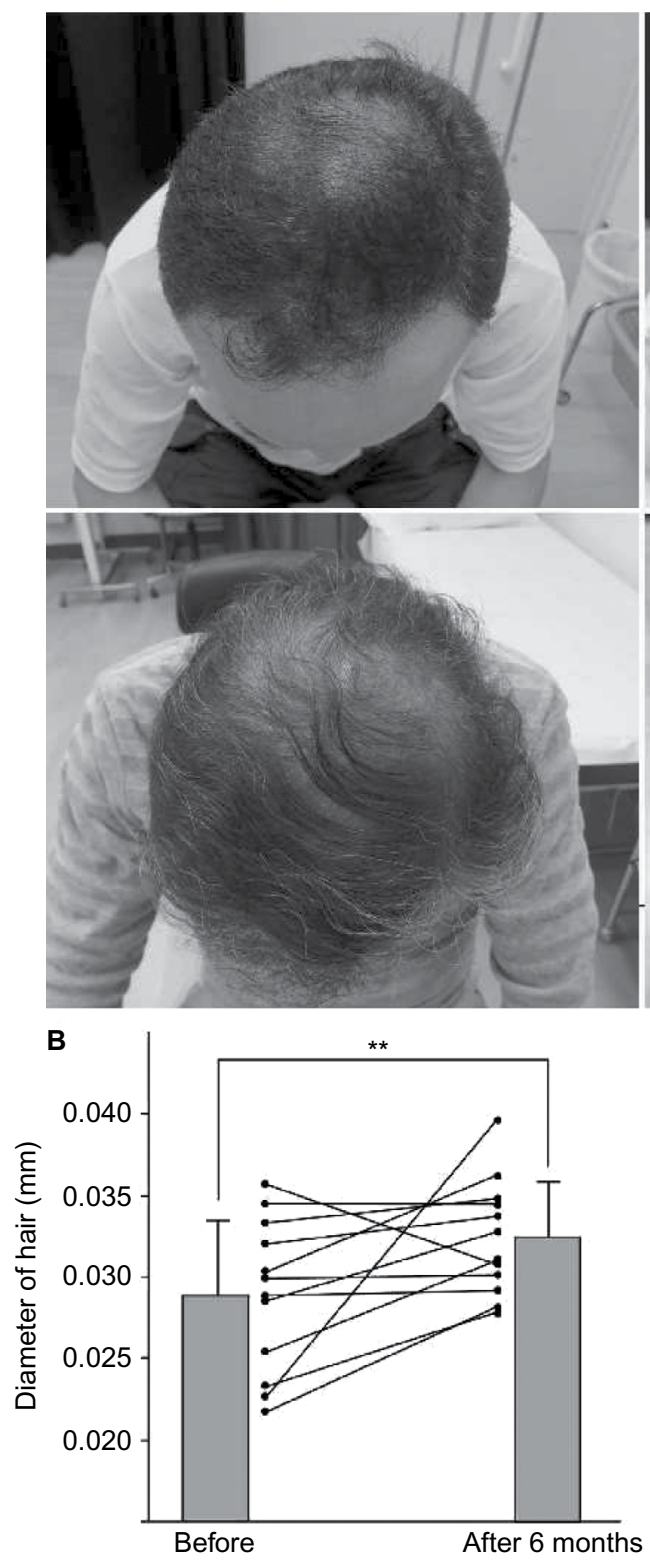

Post 6 months treatment
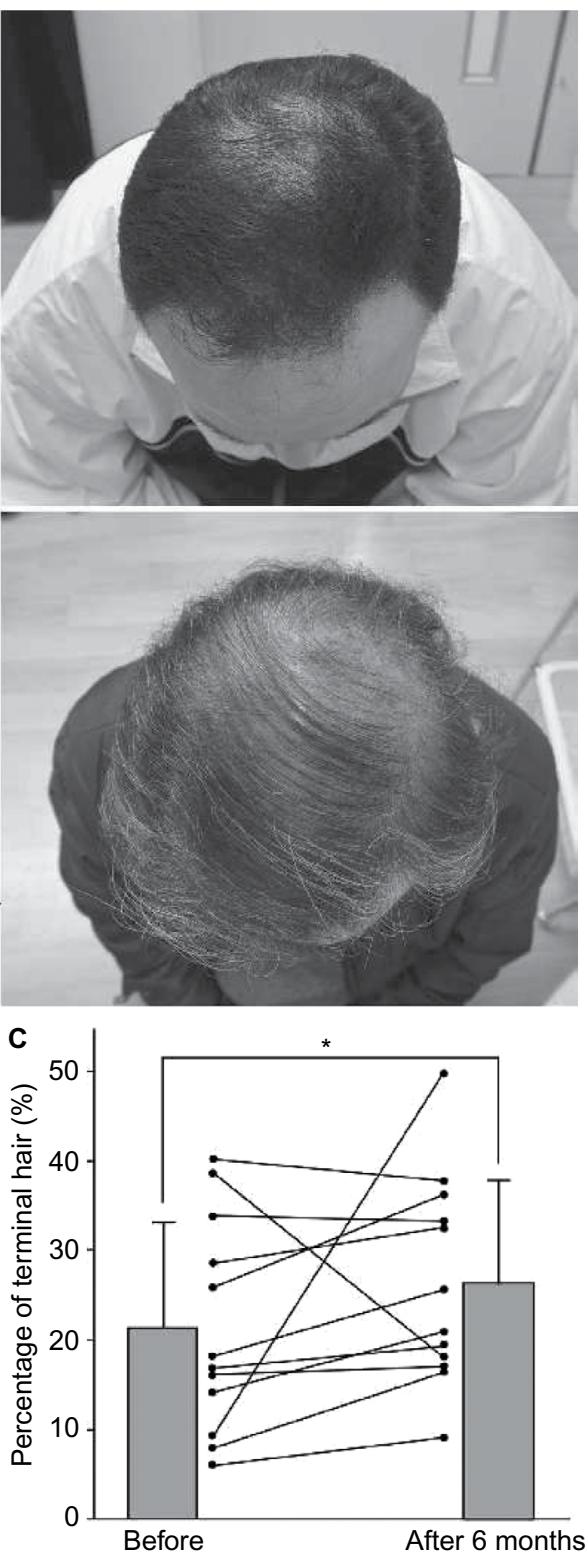

$\mathrm{N}=12,{ }^{*} P<0.05,{ }^{* *} P<0.01$, Wilcoxon signed-rank test

Figure 4 Results of the pretreatment and post 6 months of treatment.

Notes: (A) Representative photographs of improved cases. (B) Diameter of hair. (C) Percentage of terminal hair. The application of FGF-2\&D/P NPs for 6 months increased the mean diameter of hairs and the percentage of terminal hair $(n=12)$. The data represent the mean $\pm S D$. $* P<0.05$, $* * P<0.01$.

Abbreviations: D/P, dalteparin/protamine; FGF-2, fibroblast growth factor-2; NPs, nanoparticles.

compared to those without D/P NPs. Thus, FGF-2\&D/P NPs may be a stable colloidal dispersion that is suitable as an effective hair grower.

Several techniques are employed to improve the transdermal absorption of drugs. These include liposomes, hydrogels, ointments, creams, and patches incorporating various permeation enhancers. ${ }^{31}$ Recently, strategies using NPs were developed and investigated as nanomedicines to improve skin penetration. ${ }^{32,33}$ The particle size influences the functionality of the nanomedicines in terms of skin perme- ability, residence time, adherence, and degradation. The immunohistological results in this study indicate that a small part of FGF-2\&D/P NPs may penetrate into skin that has pores which are apertures of hair follicles. This is because FGF-2 immunostainings in FGF-2\&D/P NPs-applied group were higher than those of the group that received FGF-2 application without D/P NPs as well as the control groups (Figure 3).

The clinical study showed that hair growth in terms of hair diameter and percentage of terminal hair were signifi- 
cantly enhanced after the treatment with FGF-2\&D/P NPs for 6 months (Figure 4). Thus, the transdermal application of FGF-2\&D/P NPs to the scalp may be used as a new treatment for alopecia. Furthermore, because all the components of FGF-2\&D/P NPs, ie, dalteparin, protamine, and FGF-2, are in clinical use, it is likely that the FGF-2\&D/P NPs are safe for clinical use.

\section{Conclusion}

Basic and clinical research was conducted using FGF-2\&D/P NPs by itself in 12 participants (including one woman) with thin hair. The diameter, the zeta charge, and the growthstimulatory activity were stable for HMVECs in vitro of FGF-2\&D/P NPs. The hair diameter and percentage of terminal hair significantly increased after the administration of FGF-2\&D/P NPs for 6 months. The method with FGF-2\&D/P NPs is simpler and cheaper than conventional methods.

\section{Acknowledgments}

This work was funded by the National Defense Medical College in the Japanese Defense Ministry.

\section{Disclosure}

The authors report no conflicts of interest in this work.

\section{References}

1. Jain R, De-Eknamkul W. Potential targets in the discovery of new hair growth promoters for androgenic alopecia. Expert Opin Ther Targets. 2006;126:1459-1468.

2. Paus R, Cotsarelis G. The biology of hair follicles. $N$ Eng $J$ Med. 1999;341(7):491-497.

3. Wosicka H, Cal K. Targeting to the hair follicles: current status and potential. J Dermatol Sci. 2010;57(2):83-89.

4. Hwang KA, Hwang YL, Lee MH, et al. Adenosine stimulates growth of dermal papilla and lengthens the anagen phase by increasing the cysteine level via fibro blast growth factors 2 and 7 in an organ culture of mouse vibrissae hair follicles. Intern J Mole Med. 2012;29(2):195-201.

5. Jahoda CA, Horne KA, Oliver RF. Induction of hair growth by implantation of cultured dermal papilla cells. Nature. 1984;311(5986):560-562.

6. Du-Cros DL. Fibroblast growth factor and epidermal growth factor in hair development. J Invest Dermatol. 1993;101(1 Suppl):106S-113S.

7. Shi HX, Lin C, Lin BB, et al. The anti-scar effects of basic fibroblast growth factor on the wound repair in vitro and in vivo. PLoS One. 2013;8(4):e59966.

8. Lou X, Yuan H, Xie J, Wang X, Yang L, Zhang Y. Growth factors have a protective effect on neomycin-induced hair cell loss. Cell Biol Inter. 2015;39(1):65-73.

9. Ozeki M, Tabata Y. Promoted growth of murine hair follicles through controlled release of basic fibroblast growth factor. Tissue Eng. 2002;8(3):359-366.

10. Berth G, Voigh A, Dautzenberg H, Donath E, Mohwald H. Polyelectrolyte complex and layer-by layer capsules from chitosan/chitosan sulfate. Biomacromolecules. 2002;3(3):579-590.
11. Houska M, Brynda E, Bahata K. The effect of polyelectrolyte chain length on layer-by layer protein/polyelectrolyte assembly - an experimental study. J Colloid Interface Sci. 2004;273(1):140-147.

12. Hagiwara K, Kishimoto S, Ishihara M, Koyama Y, Mazda O, Sato T. In vivo gene transfer using $\mathrm{pDNA} /$ chitosan/chondroitin sulfate ternary complexes: influence of chondroitin sulfate on the stability of freezedried complexes and transfer gene expression in vivo. J Gene Med. 2013;15(2):83-92.

13. Lindahl U, Kjellen L. Pathophysiology of heparan sulfate: many diseases, few drugs. J Intern Med. 2013;273(6):5555-5571.

14. Ishihara M, Ono K. Structure and function of heparin and heparan sulfate: heparinoid library and modification of FGF-activities. Trends Glycosci Glycotechnol. 1998;10(52):223-233.

15. Hirsh J, Warkentin TE, Shaughnessy SG, et al. Heparin and lowmolecular heparin, mechanisms of action, pharmacokinetics, dosing, monitoring, efficacy, and safety. Chest. 2001;119(1 Suppl): 64S-94S.

16. Wolzt M, Wetermann A, Nieszpaur-Los M, et al. Studies on the neutralizing effects of protamine on unfractionated and low molecular weight heparin (Fragmin $\left.{ }^{\circledR}\right)$ at the site of activation of the coagulation system in man. Thromb Haemost. 1995;73(3):439-443.

17. Pan M, Lezo JS, Medina A, et al. In-laboratory removal of femoral sheath following protamine administration in patients having intracoronary stent implantation. Am J Cardiol. 1997;80(10): 1336-1338.

18. Ishihara M, Kishimoto S, Takikawa M, Hattori H, Nakamura S, Shimizu M. Biomedical application of low molecular weight heparin/protamine nano/micro particles as cell- and growth factor-carriers and coating matrix. Int J Mol Sci. 2015;16(5):11785-11803.

19. Nemeno JGE, Lee S, Yang W, Lee KM, Lee JI. Applications and implications of heparin and protamine in tissue engineering and regenerative medicine. Biomed Res Int. 2014;2014:10.

20. Mori Y, Nakamura S, Kishimoto S, et al. Preparation and characterization of low-molecular-weight heparin/protamine nanoparticles (LMW$\mathrm{H} / \mathrm{P}$ NPs) as FGF-2 carrier. Int J Nanomed. 2010;5:147-155.

21. Nakamura S, KanataniY, Kishimoto S, et al. Controlled release of FGF-2 using fragmin/protamine microparticles and effect on neovascularization. J Biomed Mater Res A. 2009;91(3):814-823.

22. Kishimoto $\mathrm{S}$, Ishihara $\mathrm{M}$, Nakamura $\mathrm{S}$, et al. Fragmin/protamine microparticles to absorb and protect HGF and to function as local HGF carrier in vivo. Acta Biomater. 2013;9(1):4763-4770.

23. Takikawa M, Nakamura SI, Nakamura S, et al. Enhancement of vascularization and granulation tissue formation by growth factors in human platelet-rich plasma containing fragmin/protamine microparticles. $J$ Biomed Mater Res B Appl Biomater. 2011;97(2):373.

24. Takikawa M, Nakamura SI, Nakamura S, et al. Enhanced effect of platelet-rich plasma containing a new carrier on hair growth. Dermatol Surg. 2011;37(12):1-9.

25. Takikawa $M$, Nakamura $S$, Ishihara $M$, et al. Improved angiogenesis and repair of tissue injury at compression sites in crush syndrome by fibroblast growth factor-2-containing Fragmin/protamine nanoparticles. J Surg Res. 2015;196(2):247-257.

26. Rosenquist TA, Martin GR. Fibroblast growth factor signaling in the hair growth cycle: expression of the fibroblast growth factor receptor and ligand genes in the murine hair follicle. Dev Dyn. 1996;205(4): 379-386.

27. Zhang X, Wang Y, Gao Y, et al. Maintenance of high proliferation and multipotent potential of human hair follicle-derived mesenchymal stem cells by growth factors. Int J Mol Med. 2013;31(4):913-921.

28. Lin WH, Xiang LJ, Shi HX, et al. Fibroblast growth factors stimulate hair growth through $\beta$-catenin and shh expression in C57BL/6 mice. Biomed Res Int. 2015;2015:730139.

29. Takikawa M, Sumi Y, Ishihara M, et al. PRP\&F/P MPs improved survival of dorsal paired pedicle skin flaps in rats. J Surg Res. 2011;170(1): e189-e196. 
30. Takabayashi Y, Ishihara M, Sumi Y, Takikawa M, Nakamura S, Kiyosawa T. Platelet-rich plasma-containing fragmin-protamine micro-nanoparticles promote epithelialization and angiogenesis in split-thickness skin graft donor sites. J Surg Res. 2015;193(1):483-491.

31. Nagai N, Iwamae A, Tanimoto S, Yoshioka C, Ito Y. Pharmacokinetics and anti-inflammatory effect of novel gel system containing ketoprofen solid nanoparticles. Biol Pharm Bull. 2015;38(12):1918-1924.
32. Osmond-McLeod MJ, Oytam Y, Kirby JK, Gomez-Fernandez L, Baxter B, McCall MJ. Dermal absorption and short-term biological impact in hairless mice from sunscreens containing zinc oxide nano- or larger particles. Nanotoxicology. 2014;8(S1):72-84.

33. Kimura E, Kawano Y, Todo H, Ikarashi Y, Sugibayashi K. Measurement of skin permeation/penetration of nanoparticles for their safety evaluation. Biol Pharm Bull. 2012;35(9):1476-1486.

\section{Publish your work in this journal}

Clinical, Cosmetic and Investigational Dermatology is an international, peer-reviewed, open access, online journal that focuses on the latest clinical and experimental research in all aspects of skin disease and cosmetic interventions. This journal is included on PubMed. The manuscript management system is completely online and includes a very quick and fair peer-review system, which is all easy to use. Visit http://www.dovepress.com/testimonials.php to read real quotes from published authors 